\title{
Dermatology and Venereology
}

\section{Pemphigus Erythematosus of the Scalp Developed Around the Postoperative Scar}

\author{
Elena Denisova, $\mathrm{MD}, \mathrm{PhD}^{1}$; Kristina Plieva, $\mathrm{MD}^{1,2}$; Maksim Bobrov, $\mathrm{MD}, \mathrm{PhD}^{1}$; \\ Vladimir Sobolev, $\mathrm{PhD}^{2,3 *}$; Irina Korsunskaya, $\mathrm{MD}, \mathrm{PhD}, \mathrm{ScD}^{2}$ \\ ${ }^{1}$ Moscow Scientific and Practical Center of Dermatology, Venerology and Cosmetology \\ ${ }^{2}$ Center of Theoretical Problems of Physico-Chemical Pharmacology, RAS \\ ${ }^{3}$ I.I. Mechnikov Research Institute for Vaccines and Sera \\ Moscow, the Russian Federation
}

\begin{abstract}
We present a clinical case reflecting the difficulties of differential diagnosis of pemphigus erythematosus (PE) in a 40-yearold male presented with erythematous topic lesions, pustules, and crusts on the skin of the scalp and face, which had existed for the previous 20 years of his life. In our case, clinical manifestations of dermatosis were atypical due to the presence of pustules and crusts impregnated with seropurulent exudates, resistant to antibiotic therapy. The patient had areas of atrophy on the scalp that could be a sign of discoid lupus erythematosus. The negative results of tests for LE cells and double-strand DNA antibodies, as well as ineffective treatment with plaquenil, antibiotics and itraconazole, helped to verify the diagnosis of PE. (International Journal of Biomedicine. 2018;8(4):358-359.)
\end{abstract}

Key Words: pemphigus erythematosus $\bullet$ differential diagnosis $\bullet$ biopsy $\bullet$ prednisolone

\section{Introduction}

Pemphigus vulgaris is an autoimmune skin disease, histologically characterized by akantholysis in epidermis. ${ }^{(1)}$ Pemphigus is classified as a rare dermatosis; its frequency is $0.5-3.2$ cases per 100,000 patients per year. Currently, there are four types of pemphigus: pemphigus vulgaris, pemphigus vegentans, pemphigus foliaceus, and pemphigus erythematosus(seborrheic) (PE). ${ }^{(2)}$ It is important to mention that PE, also known as Senear-Usher syndrome, is the overlap-syndrome with features of lupus erythematosus (LE) and pemphigus vulgaris. The clinical signs of PE are various, but generally they are presented by small, flaccid bullae with scaling and crusting. Typically, PE lesions involve the skin of the scalp, face, upper part of the chest, and back. ${ }^{(3)}$ It is necessary to remember that PE facial manifestations can be presented as a butterfly distribution on the bridge of the nose and on the malar areas, as seen in LE. ${ }^{(4)}$ It is known that skin

*Corresponding author: Vladimir Sobolev, PhD. Center of Theoretical Problems of Physico-Chemical Pharmacology, RAS Moscow, Russia.E-mail: vlsobolew@gmail.com rash on the scalp typically has features of seborrheic dermatitis, but sometimes it manifests as circumscribed lesions with adherent massive crusts and weeping followed by alopecia and cicatricial atrophy. ${ }^{(4)}$

\section{Case Presentation}

We present a clinical case reflecting the difficulties of PE differential diagnosis in a 40-year-old male presented with erythematous topic lesions, pustules, and crusts on the skin of the scalp and face, which had existed for the previous 20 years of his life. The patient considered these lesions related to a closed craniocerebral injury and subsequent neurosurgical operation with trepanation of the skull and removal of an intracranial hematoma in 1993. The skin rash first appeared at the periphery of the surgical scar. The patient applied topical antibacterial ointments without significant effect.

Observation of the skin surface of the scalp revealed massive dirty-yellow crusts impregnated with seropurulent exudate, solitary pustules, and big areas of erythema. Infiltrated pink-red papules and grey-white desquamation against the erythematous background were presented on the skin of the malar area (Figure 1). 


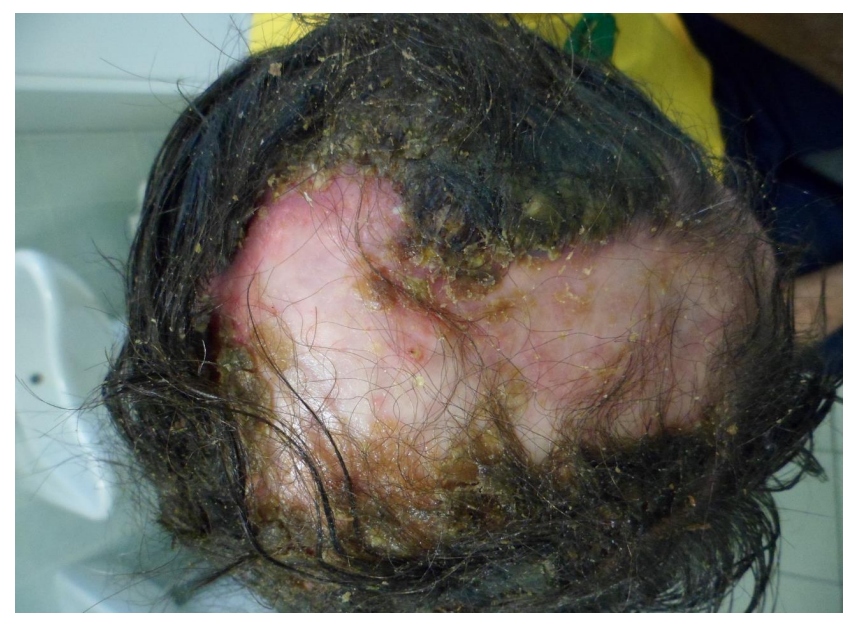

Fig. 1. Clinical manifestations of pemphigus erythematosus.

Patient received ex juvantibus therapy with plaquenil $200 \mathrm{mg} 2$ times per day for 2 weeks without significant effect. $\mathrm{He}$ was also administered subsequent antibiotic courses (doxycycline $100 \mathrm{mg} 2$ times per day for 10 days, azitromycin $250 \mathrm{mg} 2$ times per day for 10 days, and amoxicillin $500 \mathrm{mg}$ per day for one week) and itraconazole $200 \mathrm{mg} 2$ times per day for 10 days without effect.

The skin biopsy revealed focal parakeratosis, neutrophilic infiltration of stratum corneum and the upper part of stratum spinosum, as well as a lack of stratum corneum at several areas and perivascular lymphohistiocytic infiltration with the presence of eosinophils in derma (Figure 2).

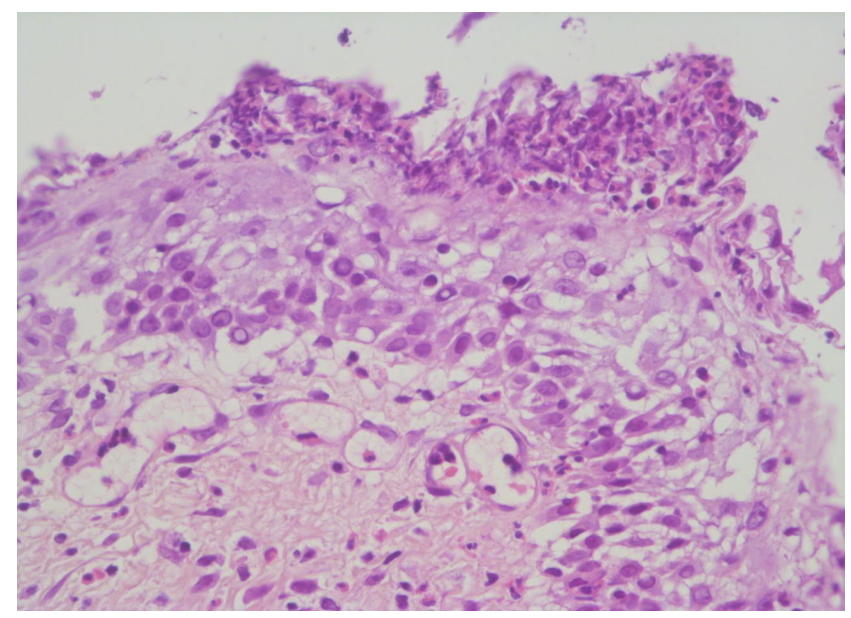

Fig. 2. Histological examination of a patient with pemphigus erythematosus.

According to the results of laboratory tests, LE cells and Demodex folliculorum were not found. The level of antidsDNA-NcX IgG in serum was $0.00 \mathrm{ME} / \mathrm{ml}(\mathrm{N}<100 \mathrm{ME} / \mathrm{ml})$.
The culture test of the pustular content demonstrated a growth of Staphylococcus aureus (+++) and Staphylococcus epidermidis $(+++)$. Due to technical difficulties, it was impossible to perform impression smear cytology to detect akantolythic cells.

According to the results of observation and laboratory tests, the patient was diagnosed with pemphigus erythematosus. He received prednisolone (starting dose $90 \mathrm{mg}$ ) with the clinical effect of the crust being gradually dried and rejected, and the number of pustules being reduced. After achieving clinical improvement, he was discharged with instructions to continue prednisolone $85 \mathrm{mg}$ with a gradual dose reduction as an outpatient.

\section{Discussion}

Diagnosis and verification of PE are difficult and require deep knowledge and experience. In our case, clinical manifestations of dermatosis were atypical due to the presence of pustules and crusts impregnated with seropurulent exudates, resistant to antibiotic therapy. It is worth mentioning that a similar case of the pustular form of PE was previously described by G.Yang et al. in 2014. ${ }^{(4)}$

The patient had areas of atrophy on the scalp that could be a sign of discoid lupus erythematosus. The negative results of tests for LE cells and double-strand DNA antibodies, as well as ineffective treatment with plaquenil, antibiotics and itraconazole, helped to verify the diagnosis of PE. However, it is important to remember that the biopsy is the most important diagnostic tool for bullous dermatoses.

\section{Conflict of interest}

The authors declare that they have no competing interests.

\section{References}

1. Bystryn JC, Rudolph JL. Pemphigus. Lancet. 2005; 366(9479):61-73.

2. James WD, Berger TG, Elston DM, Neuhaus IM. Andrews' Diseases of the Skin: Clinical Dermatology. The 12th edition. Philadelphia, PA: Elsevier; 2016.

3. Hammers CM, Schmidt E, Borradori L. Pemphigus Foliaceus and Pemphigus Erythematosus. In Katsambas AD, Lotti TM, Dessinioti C, D'Erme AM, editors. European Handbook of Dermatological Treatments. Third Edition. Springer-Verlag Berlin Heidelberg; 2015:691-700.

4. Yang GL, Zhao M, Wang JF, Xiao H, Pan Z. A Rare Presentation of Pemphigus Erythematosus as Pustules. J Clin Exp Dermatol Res. 2014;5:222. doi:10.4172/21559554.1000222. 